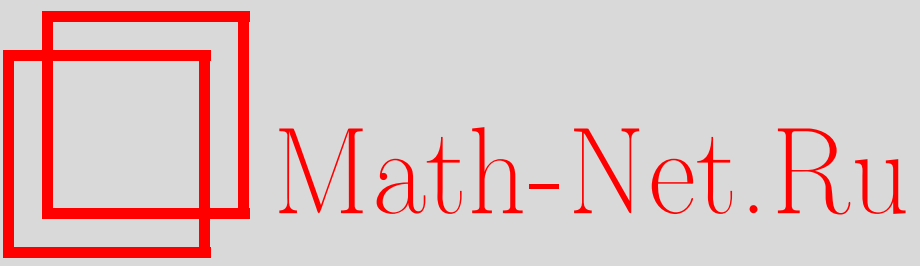

Ф. М. Мухамедов, Об алгебрах фон Неймана, соответствующих трансляционноинвариантным гиббсовским состояниям модели Изинга на решетке Бете, ТМФ, 2000, том 123, номер 1, 88-93

DOI: https://doi.org/10.4213/tmf588

Использование Общероссийского математического портала Math-Net.Ru подразумевает, что вы прочитали и согласны с пользовательским соглашением

http://www. mathnet.ru/rus/agreement

Параметры загрузки :

IP: 18.207 .199 .55

26 апреля 2023 г., 17:43:32 


\section{ОБ АЛГЕБРАХ ФОН НЕЙМАНА, СООТВЕТСТВУЮЩИХ ТРАНСЛЯЦИОННО-ИНВАРИАНТНЫМ ГИББСОВСКИМ СОСТОЯНИЯМ МОДЕЛИ ИЗИНГА НА РЕШЕТКЕ БЕТЕ}

Рассматривается модель Изинга на решетке Бете порядка $k \geqslant 2$. Для экстремальных трансляционно-инвариантных гиббсовских состояний этой модели найдены связи между алгебрами фон Неймана, порожденными при ГНС-представлении этими состояниями. Показано, что эти алгебры могут иметь типы $\mathrm{III}_{\lambda}, \lambda \in(0,1)$, и $\mathrm{III}_{1}$.

\section{1. ВВЕДЕНИЕ}

Известно [1, теорема 5.3.30], что при существовании фазового перехода в некоторой гамильтоновой системе любые два из экстремальных предельных гиббсовских состояний либо совпадают, либо дизъюнктны [2]. Поэтому естествен вопрос об алгебраической эквивалентности таких состояний, т.е. об изоморфизме алгебр фон Неймана, порожденных конструкцией Гельфанда-Наймарка-Сигала (ГНС) по таким состояниям. По-видимому, общий ответ на этот вопрос получить невозможно, но представляет интерес рассмотрение конкретных систем. Одной из них является модель Изинга, изучению которой посвящено много работ (см., например, [3-6]).

В статье [7] рассматривалась свободная решетчатая квантовая спиновая система с двумя состояниями в каждой точке, причем первое состояние занято с вероятностью $p$, а второе - с вероятностью $q=1-p$. Было показано, что соответствуюшая алгебра фон Неймана является фактором типа $\mathrm{III}_{\lambda}$, где $\lambda=p / q$.

В работе [8] изучались марковские состояния на одномерной квантовой спиновой системе, порож даемые стохастическими матрицами. Было показано, что, как правило, эти состояния порождают фактор типа $\mathrm{III}_{1}$. Однако существуют и марковские состояния, которые порождают фактор типа III $_{\lambda}$. Заметим, что модулярные свойства таких состояний были изучены в работе [9].

${ }^{*}$ Институт математики им. В.И. Романовского АН РУ, Ташкент, Республика Узбекистан. E-mail: root@im.tashkent.su 
В данной статье рассматривается модель Изинга на решетке Бете порядка $k \geqslant 2$. Для экстремальных трансляционно-инвариантных гиббсовских состояний этой модели найдены связи между алгебрами фон Неймана, порожденными при ГНС-представлении такими состояниями. Показано, что эти алгебры могут иметь типы $\mathrm{III}_{\lambda}, \lambda \in(0,1)$, и $\mathrm{III}_{1}$.

\section{2. ОПРЕДЕЛЕНИЯ И ОБОЗНАЧЕНИЯ}

Решетка Бете $\mathcal{G}^{k}$ (или дерево Кэли в другой терминологии [10]) порядка $k \geqslant 1$ есть бесконечное дерево, т.е. граф без циклов, из каждой вершины которого выходят ровно $k+1$ ребер. Пусть $\mathcal{G}^{k}=(V, \Lambda, i)$, где $V$ - множество вершин $\mathcal{G}^{k}, \Lambda$ - его множество ребер и $i$ - функция инцидентности, сопоставляюшая каждому ребру $l \in \Lambda$ его концевые точки $x, y \in V$. Если $i(l)=\{x, y\}$, то $x$ и $y$ называются соседними вериинами, и в этом случае мы будем писать, что $l=\langle x, y\rangle$. Расстояние $d(x, y), x, y \in V$, на решетке Бете вводится формулой

$$
\begin{aligned}
d(x, y)= & \min \left\{d \mid \exists x=x_{0}, x_{1}, \ldots, x_{d-1}, x_{d}=y \in V \text { такие },\right. \text { что } \\
& \text { пары } \left.\left\langle x_{0}, x_{1}\right\rangle, \ldots,\left\langle x_{d-1}, x_{d}\right\rangle-\text { ближайшие соседи }\right\} .
\end{aligned}
$$

Для произвольной точки $x^{0} \in V$ положим

$$
\begin{aligned}
W_{n} & =\left\{x \in V \mid d\left(x, x^{0}\right)=n\right\}, \\
V_{n} & =\bigcup_{m=0}^{n} W_{m}=\left\{x \in V \mid d\left(x, x^{0}\right) \leqslant n\right\}, \\
L_{n} & =\left\{l=\langle x, y\rangle \in L \mid x, y \in V_{n}\right\} .
\end{aligned}
$$

В ферромагнитной модели Изинга на решетке Бете спиновые переменные $\sigma(x), x \in V$, принимают значения \pm 1 и гамильтониан имеет вид

$$
H(\sigma)=-J \beta \sum_{\langle x, y\rangle} \sigma(x) \sigma(y),
$$

где суммирование ведется по всем парам ближайших соседей $\langle x, y\rangle$ и $J>0, \beta>0$.

Напомним некоторые факты из теории алгебр фон Неймана. Пусть $B(H)$ - алгебра всех ограниченных линейных операторов на гильбертовом пространстве $H$ (над полем комплексных чисел).

Слабо (операторная) замкнутая *-подалгебра $M$ в $B(H)$ называется алгеброй фон Неймана, если она содержит тождественный оператор 1. Алгебру фон Неймана называют фактором, если ее центр $Z(M)=\{x \in M: x y=y x, \forall y \in M\}$ тривиален, т.е. $Z(M)=\{\lambda \mathbf{1} \mid \lambda \in C\}$.

Алгебры фон Неймана разбиваются на классы I $\left(\mathrm{I}_{n}, n<\infty ; \mathrm{I}_{\infty}\right), \mathrm{II}\left(\mathrm{II}_{1} ; \mathrm{II}_{\infty}\right)$ и III.

Линейный функционал $\omega$ на $M$ называется положительныцм, если $\omega\left(x^{*} x\right) \geqslant 0$ для всех $x \in M$. Положительный функционал $\omega$ на $M$ с $\omega(\mathbf{1})=1$ называется состоянием. 
Состояние $\omega$ называется нормальнылм, если для любой ограниченной возрастаюшей сети $\left\{x_{\alpha}\right\}$ положительных элементов из $M$ выполнено условие $\omega\left(\sup _{\alpha} x_{\alpha}\right)=\sup _{\alpha} \omega\left(x_{\alpha}\right)$. Состояние $\omega$ называется следом (соответственно точным следом), если выполнено условие $\omega(x y)=\omega(y x)$ для всех $x, y \in M$ (соответственно $\left.\omega\left(x^{*} x\right)=0 \Rightarrow x=0\right)$.

Пусть $M$ - фактор, $\omega$ - точное нормальное состояние на $M$ и $\sigma_{t}^{\omega}$ - модулярная группа, ассоциированная с $\omega$ на $M$ [2, определение 2.5.15]. Через $\Gamma\left(\sigma^{\omega}\right)$ обозначим спектр Конна модулярной группы $\sigma_{t}^{\omega}[11$, определение 2.2.1].

ОПРЕДЕЛЕНИЕ [11]. Фактор $M$ имеет типы:

1) $\mathrm{III}_{1}$, если $\Gamma\left(\sigma^{\omega}\right)=R$;

2) $\operatorname{III}_{\lambda}$, если $\Gamma\left(\sigma^{\omega}\right)=\{n \ln \lambda, n \in Z\}, \lambda \in(0,1)$;

3) $\mathrm{III}_{0}$, если $\Gamma\left(\sigma^{\omega}\right)=0$.

Более подробно об алгебрах фон Неймана и модулярной теории операторных алгебр можно прочитать в книгах $[12,13]$.

\section{3. ПОСТРОЕНИЕ ТРАНСЛЯЦИОННО-ИНВАРИАНТНЫХ ГИББСОВСКИХ СОСТОЯНИЙ МОДЕЛИ ИЗИНГА}

Рассмотрим $C^{*}$-алгебру $A=\bigotimes_{\mathcal{G}^{k}} M_{2}(C)$, где $M_{2}(C)$ - алгебра матриц $2 \times 2$ над полем комплексных чисел $C$ и $k \geqslant 2$. Легко видеть [14], что квантованньй гамильтониан рассматриваемой модели Изинга имеет вид

$$
H=-\sum_{\langle x, y\rangle} \Phi_{\langle x, y\rangle}, \quad \Phi_{\langle x, y\rangle}=\left(\begin{array}{cccc}
\ln \frac{e^{\alpha}}{e^{\alpha}+e^{-\alpha}} & 0 & 0 & 0 \\
0 & \ln \frac{e^{-\alpha}}{e^{\alpha}+e^{-\alpha}} & 0 & 0 \\
0 & 0 & \ln \frac{e^{-\alpha}}{e^{\alpha}+e^{-\alpha}} & 0 \\
0 & 0 & 0 & \ln \frac{e^{\alpha}}{e^{\alpha}+e^{-\alpha}}
\end{array}\right)
$$

где $\alpha=J \beta$ и суммирование ведется по всем парам ближайших соседей $\langle x, y\rangle$. Обозначим

$$
p_{11}^{(\alpha)}=\frac{e^{\alpha}}{e^{\alpha}+e^{-\alpha}}, \quad p_{12}^{(\alpha)}=\frac{e^{-\alpha}}{e^{\alpha}+e^{-\alpha}}, \quad p_{21}^{(\alpha)}=p_{12}^{(\alpha)}, \quad p_{22}^{(\alpha)}=p_{11}^{(\alpha)} .
$$

Известно [1, п. 6.2.2], что гиббсовские состояния в конечном объеме $V_{n}$ на алгебре $A\left(V_{n}\right)=\bigotimes_{x \in V_{n}} M_{2}(C)$ определяются следуюшим образом:

$$
\omega_{V_{n}}(x)=\frac{\operatorname{tr}\left(\exp \left\{-H\left(V_{n}\right)+H\left(V_{n}, \partial V_{n}\right)\right\} x\right)}{\operatorname{tr}\left(\exp \left\{-H\left(V_{n}\right)+H\left(V_{n}, \partial V_{n}\right)\right\}\right)}, \quad x \in A\left(V_{n}\right),
$$

где

$$
H\left(V_{n}\right)=-\sum_{\langle x, y\rangle \in L_{n}} \Phi_{\langle x, y\rangle}, \quad H\left(V_{n}, \partial V_{n}\right)=\sum_{x \in W_{n}} h_{x} \sigma_{x}^{z}, \quad \sigma_{x}^{z}=\left(\begin{array}{cc}
1 & 0 \\
0 & -1
\end{array}\right)
$$

$h_{x} \in R$ - вещественнозначная функция $x \in V$. 
Из результатов статьи [5] следует, что предельные гиббсовские состояния для $\left\{\omega_{V_{n}}\right\}$ сушествуют, если функция $h_{x}$ удовлетворяет некоторому условию согласования. Трансляционно-инвариантным состояниям [14] соответствует функция $\left\{h_{x}=h=\right.$ const, $x \in V\}$, где $h$ удовлетворяет уравнению

$$
h=k \operatorname{arcth}(\theta \operatorname{th} h), \quad \theta=\operatorname{th} \alpha .
$$

При условии $1 / k<\theta<1$ сушествуют три различных решения $h=0, \pm h_{*}, h_{*}>0$ этого уравнения. В противном случае решение $h=0$ единственно. Через $\omega_{ \pm} h_{*}$ и $\omega_{0}$ обозначим соответствующие трансляционно-инвариантные состояния. Из результатов $[5,15]$ вытекает следующая теорема

ТеОрема 1. При условии $k^{-1}<\theta<k^{-1 / 2}$ все три состояния $\omega_{0} u \omega_{ \pm h_{*}}$ являются экстремальными.

Далее будет рассматриваться случай, когда все три состояния являются экстремальными гиббсовскими состояниями на множестве всех предельных гиббсовских состояний.

\section{4. АЛГЕБРЫ ФОН НЕЙМАНА, СООТВЕТСТВУЮШИЕ СОСТОЯНИЯМ $\omega_{ \pm h_{*}}$ И $\omega_{0}$ ПРИ ГНС-ПРЕДСТАВЛЕНИИ}

В этом разделе определим типы алгебр фон Неймана $\pi_{\omega_{\gamma}}(A)^{n}, \gamma=0, \pm h_{*}$, где $\pi_{\omega_{\gamma}}-$ ГНС-представления, ассоциированные с состояниями $\omega_{\gamma}\left(\gamma=0, \pm h_{*}\right)[2$, определение 2.3.18]. Из теоремы 1 и теоремы 5.3.30 в книге [1] вытекает, что состояния $\omega_{\gamma}$, $\gamma=0, \pm h_{*}$, взаимно дизъюнктны. В силу экстремальности этих состояний очевидно, что алгебры фон Неймана $\pi_{\omega_{\gamma}}(A)^{\prime \prime}, \gamma=0, \pm h_{*}$, являются факторами. Так как состояние $\omega_{0}$ марковское [16], то, рассуждая так же, как и в работе [8], получаем в силу равенств

$$
\frac{p_{11}^{(\alpha)}}{p_{12}^{(\alpha)}}=\lambda^{-1}, \quad \frac{p_{11}^{(\alpha)}}{p_{21}^{(\alpha)}}=1, \quad \frac{p_{12}^{(\alpha)}}{p_{22}^{(\alpha)}}=1, \quad \frac{p_{21}^{(\alpha)}}{p_{22}^{(\alpha)}}=\lambda,
$$

где $\lambda=e^{-2 \alpha}$, что алгебра фон Неймана $\pi_{\omega_{0}}(A)^{\prime \prime}$ является фактором типа $\mathrm{III}_{\lambda}$. Итак, справедлива следуюшая теорема.

Теорема 2. Алгебра фон Неймана $\pi_{\omega_{0}}(A)^{\prime \prime}$ является фактором типа $\mathrm{III}_{\lambda}$.

Теперь рассмотрим состояние $\omega_{ \pm h_{*}}$. Пусть $M_{ \pm h_{*}}$ обозначают факторы $\pi_{\omega_{ \pm h}}(A)^{\prime \prime}$ соответственно. Через $\sigma^{ \pm h_{*}}$ обозначим модулярные группы, ассоциированные с состояниями $\omega_{ \pm h_{*}}$, которые определяются на $M_{ \pm} h_{*}$ соответственно следуюшим образом:

$$
\begin{gathered}
\sigma_{t}^{ \pm}(x)=\lim _{n \rightarrow \infty} e^{i t \widetilde{H}\left(V_{n}\right)} x e^{-i t \widetilde{H}\left(V_{n}\right)}, \quad x \in M_{ \pm h_{*}}, \\
\widetilde{H}\left(V_{n}\right)=-H\left(V_{n}\right)+H\left(V_{n}, \partial V_{n}\right)
\end{gathered}
$$

Сушествование предела доказано в работе [1, теорема 6.2.4]. Через $M_{ \pm h_{*}}^{\sigma}$ обозначаются централизаторы $\sigma^{ \pm}$, определенные следуюшим образом:

$$
M_{ \pm h_{*}}^{\sigma}=\left\{x \in M_{ \pm h_{*}}: \sigma_{t}^{ \pm}(x)=x, \forall t \in R\right\} .
$$


Экстремальность состояний $\omega_{ \pm} h_{*}$ позволяет, так же как в [8], доказать следующую лемMy.

Лемма. Централизаторы $M_{ \pm h_{*}}^{\sigma}$ являются факторами типа $\mathrm{II}_{1}$.

Теорема 3. Если существует иелое число $p \in Z$ maкое, что $e^{h_{*}}=\lambda^{p}$, где $\lambda=$ $e^{-2 \alpha}$, то алгебры фон Неймана $M_{ \pm h_{*}}$ являются факторами типа $\mathrm{III}_{\lambda}$, в противном случае - факторами типа $\mathrm{III}_{1}$.

ДокАЗАТЕЛЬСтво. Из леммы имеем $Z\left(M_{ \pm h_{*}}^{\sigma}\right)=C \mathbf{1}$. Согласно предложению 2.2 .2 из [11] получим $\Gamma\left(\sigma^{ \pm}\right)=\operatorname{Sp}\left(\sigma^{ \pm}\right)$, где $\operatorname{Sp}\left(\sigma^{ \pm}\right)$- спектр Арвесона $[11,13]$. Если положить

$$
\sigma_{t}^{ \pm, n}=e^{i t \widetilde{H}\left(V_{n}\right)} x e^{-i t \widetilde{H}\left(V_{n}\right)}
$$

TO

$$
\operatorname{Sp}\left(\sigma^{ \pm, n}\right)=\operatorname{Sp}\left(\widetilde{H}\left(V_{n}\right)\right)-\operatorname{Sp}\left(\widetilde{H}\left(V_{n}\right)\right) \equiv\left\{\lambda-\mu: \lambda, \mu \in \operatorname{Sp}\left(\widetilde{H}\left(V_{n}\right)\right)\right\} .
$$

Из вида $H\left(V_{n}\right), H\left(V_{n}, \partial V_{n}\right)$ и равенств (1) можно легко получить, что

$$
\operatorname{Sp}\left(\sigma^{ \pm, n}\right)=\left\{m \ln \lambda+2 k h_{*}\right\}_{m=-q, k=-l}^{q, l}
$$

где $q, l$ - числа, зависящие от $n$. Отсюда, если существует такое целое число $p \in Z$, в котором $e^{h_{*}}=\lambda^{p}$, из соотношения (2) вытекает, что

$$
\operatorname{Sp}\left(\sigma^{ \pm}\right)=\{n \ln \lambda\}_{n \in Z}
$$

Следовательно, факторы $M_{ \pm h_{*}}$ имеют тип $\mathrm{III}_{\lambda}$. В противном случае имеем $\Gamma\left(\sigma^{ \pm}\right)=R$, т.е. получаем, что $M_{ \pm} h_{*}$ являются факторами типа $\mathrm{III}_{1}$. Теорема доказана.

Из единственности гиперфинитных факторов $\mathrm{III}_{\lambda}$ и $\mathrm{III}_{1}$ в силу доказанной теоремы вытекает, что факторы $M_{h_{*}}$ и $M_{-h_{*}}$ изоморфны. При условии $e^{h_{*}}=\lambda^{p}, p \in Z$, факторы $M_{0}=\pi_{\omega_{0}}(A)^{\prime \prime}$ и $M_{ \pm h_{*}}$ также изоморфны. В противном случае факторы $M_{0}$ и $M_{ \pm h_{*}}$ неизоморфны. 


\section{Список литературы}

[1] O. Bratteli, D. Robinson. Operator algebras and Quantum Statistical Mechanics. II. Berlin: Springer-Verlag, 1981.

[2] У. Брателли, Д. Робинсон. Операторные алгебры и квантовая статистическая механика. I. М.: Мир, 1982.

[3] Р. Л. Добрушин. Теор. вероятн. и ее примен. 1968. Т. 13. № 2. С. 201-229.

[4] C. M. Series, Ya. G. Sinai. Commun. Math. Phys. 1990. V. 128. P. 63-76.

[5] П. М. Блехер, Н. Н. Ганиходжаев. Теор. вероятн. и ее примен. 1990. Т. 35. № 2. С. 220-230.

[6] Н. Н. Ганиходжсаев, У. А. Розиков. ТМФ. 1997. Т. 111. № 1. С. 109-117.

[7] R. Powers. Ann. Math. 1967. V. 81. P. 138-171.

[8] N. N. Ganikhodjaev, F. M. Mukhamedov. Methods Funct. Anal. Topol. 1998. V. 4. № 3. P. 33-38.

[9] В. Я. Голодец, Г. Н. Жолткевич. ТМФ. 1983. Т. 56. № 1. С. 80-86.

[10] Р. Бәкстер. Точно решаемые модели в статистической физике. М.: Мир, 1985.

[11] A. Connes. Ann. Sci. Ec. Norm. Super. IV. Ser. 6. 1973. P. 133-252.

[12] S. Stratila, L. Zsido. Lectures on von Neumann algebras. Bucuresti: Editura Academici \& Abacus Press, 1979.

[13] S. Stratila. Modular theory in operator algebras. Bucuresti: Abacus Press, 1981.

[14] Я. Г. Синай. Теория фазовых переходов. Строгие результаты. М.: Наука, 1980.

[15] P. M. Bleher. Commun. Math. Phys. 1990. V. 128. P. 411-419.

[16] Л. Аккарди. Функ. анализ и его прилож. 1975. Т. 8. № 1. С. 1-8.

Поступила в редакцию 13.VII.1999 г. 